\title{
A NONREGULAR SOLUTION OF THE NONLINEAR DYNAMIC DISTURBANCE DECOUPLING PROBLEM WITH AN APPLICATION TO A COMPLETE SOLUTION OF THE NONLINEAR MODEL MATCHING PROBLEM*
}

\author{
H. J. C. HUIJBERTS $\dagger$
}

\begin{abstract}
The nonregular dynamic disturbance decoupling problem for nonlinear control systems is introduced. A local solution is given by means of a constructive algorithm that is based on Singh's algorithm and the clamped dynamics algorithm. Further studied is the nonlinear model matching problem that is defined as follows: given a nonlinear control system, to be referred to as the plant, and another nonlinear control system, to be referred to as the model, can a compensator for the plant be found in such a way that the input-output behavior of the compensated plant matches that of the model? By proving that the solvability of the nonlinear model matching problem is equivalent to the solvability of an associated nonregular dynamic disturbance decoupling problem, a complete local solution of this problem can be established.
\end{abstract}

Key words. nonlinear control systems, dynamic disturbance decoupling, dynamic precompensation, clamped dynamics algorithm, nonlinear model matching

AMS(MOS) subject classifications. 93C10, 93B50, 93C35

1. Introduction. Consider a nonlinear multi-input multi-output control system with disturbances, of the form

$$
\begin{aligned}
& \dot{x}=f(x)+g(x) u+p(x) q, \\
& y=h(x),
\end{aligned}
$$

where $x \in \mathscr{X}$, an open subset of $\mathbb{R}^{n}$, the inputs $u \in \mathbb{R}^{m}$, the outputs $y \in \mathbb{R}^{p}$, the disturbances $q \in \mathbb{R}^{r}, f$ and $h$ are vector-valued analytic functions, and $g$ and $p$ are matrix-valued analytic functions, all of appropriate dimensions. In the disturbance decoupling problem (DDP) for the system (1) we search for a regular static state feedback

$$
u=\alpha(x)+\beta(x) v,
$$

with $v$ a new $m$-dimensional control and $\beta(x)$ a nonsingular $m \times m$ matrix for all $x$, so that in the feedback-modified dynamics

$$
\dot{x}=f(x)+g(x) \alpha(x)+g(x) \beta(x) v+p(x) q
$$

the disturbances $q$ do not affect the outputs $y$. A local solution of the DDP using differential geometric tools was initiated in [21] and [14] and has led to a more or less complete understanding of this problem; see, e.g., [20], [33]. The nonlinear DDP forms a direct generalization of the linear DDP and the theory about the nonlinear DDP typically extends the well-known linear geometric theory (see [36]) to a nonlinear context.

The purpose of this paper is to study, as in [17], a dynamic version of the DDP for the nonlinear system (1). That is, instead of a static feedback law (2) we allow for a dynamic state feedback

$$
\begin{aligned}
& \dot{z}=\alpha(x, z)+\beta(x, z) v, \\
& u=\gamma(x, z)+\delta(x, z) v,
\end{aligned}
$$

* Received by the editors June 4, 1990; accepted for publication (in revised form) January 17, 1991.

$\dagger$ Department of Applied Mathematics, University of Twente, P.O. Box 217, 7500 AE Enschede, the Netherlands. Present address, Department of Mathematics and Computing Science, Eindhoven University of Technology, P.O. Box 513, 5600 MB Eindhoven, the Netherlands. 
with $z$ the $\mu$-dimensional compensator state, and $v$ an $m$-dimensional new control. In the dynamic version of the dynamic disturbance decoupling problem we require that in the modified dynamics

$$
\begin{aligned}
\dot{x} & =f(x)+g(x) \gamma(x, z)+g(x) \delta(x, z) v+p(x) q, \\
\dot{z} & =\alpha(x, z)+\beta(x, z) v
\end{aligned}
$$

the disturbances $q$ do not influence the outputs $y$. In [17] a regular version of this problem was studied, where regularity means that we demand the system (4) with inputs $v$ and outputs $u$ to be invertible for all $x$. We refer to this problem as the dynamic disturbance decoupling problem (DDDP). In this paper we solve a version of the DDDP where we drop the requirement of regularity of the compensator (4). This problem will be referred to as the nonregular dynamic disturbance decoupling problem (nDDDP). The reason for studying this problem is that if the DDDP is not solvable, we may still be able to solve the nDDDP, albeit at the expense of some of the effective controls. The solution is given by means of an algorithm based on the clamped dynamics algorithm (cf. [22], [35]) and Singh's algorithm (cf. [34], [9]). Basically, the method used in this paper to solve the nDDDP is an extension of the method given in [17] to solve the DDDP. Here the basic tool was Singh's algorithm. One special feature of the algorithm is that it is constructive. A drawback is that it does not give a compensator of minimal order. Therefore we cannot make any statement about the solvability of the nonregular disturbance decoupling problem by means of static state feedback.

The solution of the nDDDP presented in this paper also turns out to be of use for solving another synthesis problem: the nonlinear model matching problem (MMP). This problem can be formulated as follows: Given an affine nonlinear control system, to be referred to as the plant $P$, and another affine nonlinear control system, to be referred to as the model $M$, can we find a compensator for $P$ such that the input-output behavior of the precompensated plant matches that of $M$ ?

For linear systems this problem is completely solved (see, e.g., [11], [27], [30][32]). For nonlinear systems until now only partial solutions have appeared (see, e.g., [6], [7], [10], [15], [18], [19], [29]). It will be shown in this paper that the nonlinear MMP can be formulated as an nDDDP with disturbance measurements. This observation was already made for linear systems in [32], [11]. In [10] sufficient conditions for solvability of the nonlinear MMP were given by solving an associated DDP with disturbance measurements by means of regular static state feedback. Furthermore, the sufficient conditions for solvability of the nonlinear MMP that were given in [29] can be viewed as following from the solution of an associated DDDP with disturbance measurements by means of regular dynamic state feedback as was also studied in [17]. It is important to note that the partial solutions to the MMP mentioned above are all given in terms of structural invariants of the system under consideration, whereas the complete solution given in this paper is in terms of an algorithm.

The paper is organized as follows. In $\S 2$ we give the clamped dynamics algorithm. This algorithm allows us to determine the clamped dynamics of a nonlinear control system, i.e., the internal dynamics that are compatible with the constraint that the output is zero for all times. Furthermore, we give an important proposition that gives a connection between the clamped dynamics of a nonlinear control system and the clamped dynamics of the same system precompensated by a dynamic state feedback. In $\S 3$ we introduce the nonregular dynamic disturbance decoupling problem with disturbance measurements (nDDDPdm) and the nDDDP, and we present an algorithm for solving both problems. In $\S 4$ we formulate the nonlinear MMP and give a complete 
local solution to this problem by associating an nDDDPdm with it. In $\S 5$, finally, some conclusions will be drawn.

2. The clamped dynamics algorithm. In this section we introduce the notion of clamped dynamics and an algorithm to compute these, following [35]. Consider a nonlinear control system of the form

$$
\begin{aligned}
& \dot{x}=f(x)+g(x) u, \\
& y=h(x),
\end{aligned}
$$

where $x \in \mathscr{X}$, an open subset of $\mathbb{R}^{n}$, the inputs $u \in \mathbb{R}^{m}$, the outputs $y \in \mathbb{R}^{p}, f$ and $h$ are vector-valued analytic functions, and $g$ is a matrix-valued analytic function, all of appropriate dimensions. The clamped dynamics of (6) are defined as the internal dynamics of (6) that are compatible with the constraint that the output is zero for all times. The notion of clamped dynamics was first identified, in the single-input singleoutput case, in [4], [28] (note, however, that in these references the notion was called zero dynamics). For the multi-input multi-output case it was further elaborated in [5], [22], [35]. In [22], a general algorithm to calculate the clamped dynamics was proposed. This algorithm was based on a modified version of Hirschorn's structure algorithm (see [13]). A different (but equivalent) algorithm was proposed in [35] and is based upon a modified version of Krener's algorithm (see [25]). In this paper we follow [35].

DEFINITION 2.1. A submanifold $N \subset \mathscr{X}$ is controlled invariant for (6) if there exists an analytic feedback $u=\alpha(x)$ such that the vector field $f(x)+g(x) \alpha(x)$ is tangent to $N$.

Remark 2.2. If $\operatorname{dim}(T N+\mathscr{G})=$ constant, where $\mathscr{G}$ is the distribution spanned by the columns of $g$, this definition is equivalent to the following characterization: $N$ is controlled invariant if for any $x_{0} \in N$ there exists an admissible control $\bar{u}(t)$ such that the solution of $\dot{x}(t)=f(x)+g(x) \bar{u}(t), x(0)=x_{0}$ remains in $N$ (see [33]).

DEFINITION 2.3. $N \subset \mathscr{X}$ is an output-nulling controlled invariant submanifold for (6) if there exists a feedback $u=\alpha(x)$, such that $f(x)+g(x) \alpha(x)$ is tangent to $N$ and $h(x)$ is zero on $N$.

To determine the dynamics compatible with the constraints $y(t)=0$ for all $t$, we need to compute the maximal output-nulling controlled invariant submanifold, provided it exists. This can be done by means of the following algorithm that is based on the algorithm from [35].

Algorithm 2.4. Clamped dynamics algorithm. Consider the system (6). Let $x_{0} \in \mathscr{X}$ be such that $h\left(x_{0}\right)=0$ and assume that $h=\left(h_{1}, \cdots, h_{p}\right)$ has constant rank $p$ in a neighborhood of $x_{0}$ in $h^{-1}\left(x_{0}\right)$.

- Step 0

Locally around $x_{0}$ the set $N_{0}=h^{-1}(0)$ is an $\left(n-p_{0}\right)$-dimensional submanifold, where $p_{0}:=p$. Denote $\phi_{0}:=h$.

- Step $k$

Let $N_{k-1}$ be a smooth $\left(n-p_{k-1}\right)$-dimensional manifold through $x_{0}$, given as $N_{k-1}=\left\{x \mid \phi_{k-1}(x)=0\right\}$. Calculate

$$
\dot{\phi}_{k-1}=\frac{\partial \phi_{k-1}}{\partial x}[f(x)+g(x) u]=: A_{k}(x)+B_{k}(x) u .
$$

Assume that $B_{k}(x)$ has constant rank $r_{k}$ in a neighborhood of $x_{0}$ in $N_{k-1}$. After a possible permutation of the entries of $\phi_{k-1}$ we may assume that the first $r_{k}$ 
rows of $B_{k}$ are linearly independent. Accordingly, we write (7) as

$$
\left(\begin{array}{c}
\dot{\phi}_{\tilde{k}} \\
\dot{\phi}_{\hat{k}}
\end{array}\right)=\left(\begin{array}{c}
\tilde{A}_{k}(x)+\tilde{B}_{k}(x) u \\
\hat{A}_{k}(x)+\hat{B}_{k}(x) u
\end{array}\right)
$$

where $\tilde{B}_{k}(x)$ has full row rank $r_{k}$ in a neighborhood of $x_{0}$ in $N_{k-1}$. Let $\tilde{B}_{k}^{+}(x)$ be a right inverse of $\tilde{B}_{k}(x)$. Letting $u=-\tilde{B}_{k}^{+}(x) \tilde{A}_{k}(x)$, we find from (8) that

$$
\left(\begin{array}{c}
\dot{\phi}_{\tilde{k}} \\
\dot{\phi}_{\hat{k}}
\end{array}\right)=\left(\begin{array}{c}
0 \\
\hat{A}_{k}(x)-\hat{B}_{k}(x) \tilde{B}_{k}^{+}(x) \tilde{A}_{k}(x)
\end{array}\right)=:\left(\begin{array}{c}
0 \\
\bar{\phi}_{k}(x)
\end{array}\right) .
$$

Note that, since each row. of $\hat{B}_{k}(x)$ is linearly dependent on the rows of $\tilde{B}_{k}(x)$, $\bar{\phi}_{k}(x)$ is independent of the choice of $\tilde{B}_{k}^{+}(x)$. Assume that $\bar{\phi}_{k}\left(x_{0}\right)=0$ and that $\bar{\phi}_{k}(x)$ has constant rank $s_{k}$ in a neighborhood of $x_{0}$ in $N_{k-1}$. Then locally around $x_{0}, \quad N_{k}:=\left\{x \in N_{k-1} \mid \bar{\phi}_{k}(x)=0\right\}$ is an $\left(n-p_{k}\right)$-dimensional submanifold, with $p_{k}:=p_{k-1}+s_{k}$. Permute the entries of $\bar{\phi}_{k}$ such that the first $s_{k}$ entries are independent on $N_{k}$, and denote $\phi_{k}:=\left(\phi_{k-1}, \bar{\phi}_{k 1}, \cdots, \bar{\phi}_{k s_{k}}\right)$.

If, at every step of the algorithm, the two constant rank assumptions are satisfied and $\bar{\phi}_{k}\left(x_{0}\right)=0$, then we call $x_{0}$ a regular point for the algorithm. If $x_{0}$ is a regular point for the clamped dynamics algorithm, then it easily follows that the algorithm terminates after $k^{*}<n$ iterations, where $k^{*}$ is the least integer such that $N_{k^{*}-1}=N_{k^{*}}$, or equivalently $k^{*}$ is the least integer for which $\bar{\phi}_{k} \equiv 0$ on $N_{k-1}$. We will call the maximal connected component of $N_{k^{*}}$ containing $x_{0}$ the clamped dynamics manifold of (6) and we denote it by $N^{*}$. A control that renders the dynamics on $N^{*}$ invariant is given by $u=-\tilde{B}_{k^{*}}^{+}(x) \tilde{A}_{k^{*}}(x)$.

In what follows we will control nonlinear systems of the form (6) by means of a compensator $R$ of the form

$$
R\left\{\begin{array}{l}
\dot{z}=\alpha(x, z)+\beta(x, z) v \\
u=\gamma(x, z)+\delta(x, z) v
\end{array}\right.
$$

with $z \in \mathbb{R}^{\mu}, v$ denoting the new inputs and real analytic $\alpha, \beta, \gamma, \delta$. A question that arises is: what is the connection between the clamped dynamics manifold of (6) and the clamped dynamics manifold of (6), (10)? The following proposition gives an answer to this question.

Proposition 2.5. Let the clamped dynamics manifold of (6) be given by $N^{*}=$ $\left\{x \mid \phi_{k^{*}}(x)=0\right\}$. Then the clamped dynamics manifold of $(6),(10)$ is given by $M^{*}=$ $\left\{(x, z) \mid \phi_{k^{*}}(x)=0, \psi(x, z)=0\right\}$ for some vector of functions $\psi(x, z)$.

The proof appears in the Appendix.

3. The nonregular dynamic disturbance decoupling problem (nDDDP). In this section we formulate two kinds of nDDDPs and give a local solution for both problems.

Let $\Sigma$ be a nonlinear multi-input multi-output control system with disturbances of the form

$$
\Sigma\left\{\begin{array}{l}
\dot{x}=f(x)+g(x) u+p(x) q \\
y=h(x),
\end{array}\right.
$$

where $x \in \mathscr{X}$, an open subset of $\mathbb{R}^{n}$, the inputs $u \in \mathbb{R}^{m}$, the outputs $y \in \mathbb{R}^{p}$, the disturbances $q \in \mathbb{R}^{r}, f$ and $h$ are vector-valued analytic functions, and $g$ and $p$ are matrix-valued analytic functions, all of appropriate dimensions. Analogously to [17] we define the following problems for $\Sigma$. 
Definition 3.6. Consider the system $\Sigma$ and let a point $x_{0} \in \mathscr{X}$ be given.

1. The local nDDDP with disturbance measurements (nDDDPdm) consists in finding (if possible) a compensator $Q$ of the form

$$
Q\left\{\begin{array}{l}
\dot{z}=\alpha(x, q, z)+\beta(x, q, z) v \\
u=\gamma(x, q, z)+\delta(x, q, z) v
\end{array}\right.
$$

with $z \in \mathbb{R}^{\mu}$, a neighborhood $U$ of $x_{0}$ in $\mathscr{X}$, an open subset $V$ of $\mathbb{R}^{\mu}$, and a map $F: U \mapsto V$ with the property that $y^{\Sigma^{\circ} Q}(\bar{x}, F(\bar{x}), t)$ is independent of $q$ for all $\bar{x} \in U$. Here $y^{\Sigma \circ Q}(\bar{x}, F(\bar{x}), t)$ denotes the output of the precompensated system $\Sigma \circ Q$ initialized at $(\bar{x}, F(\bar{x}))$, at time $t$.

2. The local nDDDP consists in finding (if possible) a compensator $R$ of the form

$$
R\left\{\begin{array}{l}
\dot{z}=\alpha(x, z)+\beta(x, z) v \\
u=\gamma(x, z)+\delta(x, z) v
\end{array}\right.
$$

with $z \in \mathbb{R}^{\mu}$, a neighborhood $U$ of $x_{0}$ in $\mathscr{X}$, an open subset $V$ of $\mathbb{R}^{\mu}$, and a map $F: U \mapsto V$ with the property that $y^{\Sigma^{\circ} Q}(\bar{x}, F(\bar{x}), t)$ is independent of $q$ for all $\bar{x} \in U$.

If we require the compensators $Q$ and $R$ to be invertible, the above problems will be referred to by DDDPdm and DDDP, respectively.

A solution of the DDDP and the DDDPdm can be found in [17]. Recall that for linear systems the conditions for solvability of nDDPdm, DDPdm, nDDDPdm, and DDDPdm are all equivalent (see, e.g., [1], [2]). From Example 3.2 in [17] it has become clear that for nonlinear systems solvability of the DDP is not equivalent to the solvability of the DDDP. The following example illustrates that for nonlinear systems solvability of the nDDDP is not equivalent to solvability of the DDP nor the DDDP.

Example 3.7. Consider the nonlinear system

$$
\begin{aligned}
& \dot{x}_{1}=x_{2} u, \\
& \dot{x}_{2}=x_{3}+q, \\
& \dot{x}_{3}=x_{1} u, \\
& y_{1}=x_{1}, \\
& y_{2}=x_{3} .
\end{aligned}
$$

It is easy to see that we can solve the nDDDP for this system by putting $u=0$, while using the theory developed in [17] it can be shown that the DDDP is not solvable for (14), which also implies that the DDP is not solvable for (14).

In what follows we will give a constructive algorithm to solve the nDDDPdm. In order to get a better insight into how the algorithm works, we first discuss the ideas behind the algorithm. So, consider a system $\Sigma$ with disturbances, of the form (11). Choose a point $x_{0} \in \mathscr{X}$. First assume that we want to solve the nDDDPdm around $x_{0}$. Clearly, a compensator $Q$ solves the nDDDPdm for $\Sigma$ around $x_{0}$ if and only if for $\Sigma \circ Q$ the $y_{i}^{(j)}(t)$ do not depend on $q$ for all $t$. We now apply the first steps of Singh's algorithm (cf. [34], [9]) to $\Sigma$. Thus, for $\Sigma$ we determine

$$
\dot{y}=\frac{\partial h}{\partial x}(x)[f(x)+g(x) u+p(x) q]=: a_{1}(x)+b_{1}(x) u+c_{1}(x) q .
$$


Assume that $b_{1}(x)$ has constant rank $\rho_{1}$ in a neighborhood of $x_{0}$. After a possible permutation of the entries of $y$, we may assume that the first $\rho_{1}$ rows of $b_{1}(x)$ are linearly independent. Accordingly, we write (15) as

$$
\left(\begin{array}{c}
\dot{\tilde{y}}_{1} \\
\dot{\hat{y}}_{1}
\end{array}\right)=\left(\begin{array}{c}
\tilde{a}_{1}(x)+\tilde{c}_{1}(x) q \\
\hat{a}_{1}(x)+\hat{c}_{1}(x) q
\end{array}\right)+\left(\begin{array}{c}
\tilde{b}_{1}(x) \\
\hat{b}_{1}(x)
\end{array}\right) u,
$$

where $\tilde{b}_{1}(x)$ has full row rank $\rho_{1}$ in a neighborhood of $x_{0}$. Let $\tilde{b}_{1}^{+}(x)$ be a right inverse of $\tilde{b}_{1}(x)$. Then from the upper part of (16) it follows that

$$
u=\tilde{b}_{1}^{+}(x)\left(\dot{\tilde{y}}_{1}-\tilde{a}_{1}(x)-\tilde{c}_{1}(x) q\right) .
$$

Combining (16) and (17) we obtain

$$
\dot{\hat{y}}_{1}=\hat{a}_{1}(x)+\hat{b}_{1}(x) \tilde{b}_{1}^{+}(x)\left(\dot{\tilde{y}}_{1}-\tilde{a}_{1}(x)\right)+\left(\hat{c}_{1}(x)-\hat{b}_{1}(x) \tilde{b}_{1}^{+}(x) \tilde{c}_{1}(x)\right) q=: \psi_{1}\left(x, q, \dot{\tilde{y}}_{1}\right) .
$$

Note that $\psi_{1}$ is affine in $q$ and $\dot{\tilde{y}}_{1}$. Define the matrix-valued function

$$
D_{1}(x):=\frac{\partial \psi_{1}\left(x, q, \dot{\dot{y}_{1}}\right)}{\partial q} .
$$

Assume that $D_{1} \not \equiv 0$. This implies that $\dot{\tilde{y}}_{1}$ explicitly depends on $q$. Furthermore, this dependence is intrinsic, meaning that with another partition $\left(\tilde{y}_{1}, \hat{y}_{1}\right)$ of $y$ also a $q$-dependence would occur via a matrix-valued function $\tilde{D}_{1}(x)$ with the property that $\tilde{D}_{1}(x)=T_{1}(x) D_{1}(x)$, where $T_{1}(x)$ is invertible (cf. [9]). Using similar arguments as in the proof of Proposition 2.5, we can show that this $q$-dependence can only be resolved by means of dynamic compensation if we can choose the compensator and its initial conditions in such a way that $D_{1}(x(t))=0$ for all $t$. However, this would imply that the nDDDPdm is not solvable in a neighborhood of $x_{0}$ in $\mathscr{X}$, but at most in a neighborhood of $x_{0}$ in $\left\{x \mid D_{1}(x)=0\right\}$. Thus we must necessarily have that $D_{1} \equiv 0$ if we want to solve the nDDDPdm. Assuming that $D_{1} \equiv 0$ we proceed by determining

$$
\begin{aligned}
\hat{y}_{1}^{(2)} & =\frac{\partial \psi_{1}}{\partial x}\left(x, \dot{\tilde{y}}_{1}\right)[f(x)+g(x) u+p(x) q]+\frac{\partial \psi_{1}}{\partial \dot{\tilde{y}}_{1}}\left(x, \dot{\tilde{y}}_{1}\right) \tilde{y}_{1}^{(2)} \\
& =: a_{2}\left(x, \dot{\tilde{y}}_{1}, \tilde{y}_{1}^{(2)}\right)+b_{2}\left(x, \dot{\dot{y}_{1}}\right) u+c_{2}\left(x, \dot{\tilde{y}}_{1}\right) q .
\end{aligned}
$$

Define $B_{2}:=\left(\tilde{b}_{1}^{T} b_{2}^{T}\right)^{T}$ and assume that $B_{2}\left(x, \dot{\tilde{y}}_{1}\right)$ has constant rank $\rho_{2}$ in a neighborhood of $x_{0}$. Then, after a possible permutation of the entries of $\hat{y}_{1}$, we may assume that the first $\rho_{2}-\rho_{1}$ rows of $b_{2}\left(x, \dot{\tilde{y}}_{1}\right)$ are linearly independent. Accordingly, we write $(20)$ and the upper part of (16) as

$$
\left(\begin{array}{c}
\dot{\tilde{y}}_{1} \\
\tilde{y}_{2}^{(2)} \\
\hat{y}_{2}^{(2)}
\end{array}\right)=\left(\begin{array}{c}
\tilde{a}_{1}(x)+\tilde{c}_{1}(x) q \\
\tilde{a}_{2}\left(x, \dot{\tilde{y}}_{1}, \tilde{y}_{1}^{(2)}\right)+\tilde{c}_{2}\left(x, \dot{\tilde{y}}_{1}\right) q \\
\hat{a}_{2}\left(x, \dot{\tilde{y}}_{1}, \tilde{y}_{1}^{(2)}\right)+\hat{c}_{2}\left(x, \dot{\tilde{y}}_{1}\right) q
\end{array}\right)+\left(\begin{array}{c}
\tilde{b}_{1}(x) \\
\tilde{b}_{2}\left(x, \dot{\tilde{y}}_{1}\right) \\
\hat{b}_{2}\left(x, \dot{\tilde{y}}_{1}\right)
\end{array}\right) u,
$$

where $\tilde{B}_{2}\left(x, \dot{\tilde{y}}_{1}\right):=\left(\tilde{b}_{1}^{T}(x) \tilde{b}_{2}^{T}\left(x, \dot{\tilde{y}}_{1}\right)\right)^{T}$ has full row rank $\rho_{2}$. Let $\tilde{B}_{2}^{+}\left(x, \dot{\tilde{y}}_{1}\right)$ be a right inverse of $\tilde{B}_{2}\left(x, \dot{\tilde{y}}_{1}\right)$ and define $\tilde{Y}_{2}:=\left(\dot{\tilde{y}}_{1}^{T} \tilde{y}_{2}^{(2) T}\right)^{T}, \tilde{A}_{2}\left(x, \dot{\tilde{y}}_{1}, \tilde{y}_{1}^{(2)}\right):=\left(\tilde{a}_{1}^{T}(x) \tilde{a}_{2}^{T}\left(x, \dot{\tilde{y}}_{1}, \tilde{y}_{1}^{(2)}\right)\right)^{T}$, $\tilde{C}_{2}\left(x, \dot{\tilde{y}}_{1}\right):=\left(\tilde{c}_{1}^{T}(x) \tilde{c}_{2}^{T}\left(x, \dot{\tilde{y}}_{1}\right)\right)^{T}$. Then again we can rewrite $\hat{y}_{2}^{(2)}$ as

$$
\begin{aligned}
\hat{y}_{2}^{(2)}= & \hat{a}_{2}\left(x, \dot{\dot{y}_{1}}, \tilde{y}_{1}^{(2)}\right)-\hat{b}_{2}\left(x, \dot{\tilde{y}}_{1}\right) \tilde{B}_{2}^{+}\left(x, \dot{\dot{y}_{1}}\right)\left(\tilde{A}_{2}\left(x, \dot{\tilde{y}}_{1}, \tilde{y}_{1}^{(2)}\right)-\tilde{Y}_{2}\right) \\
& +\left(\hat{c}_{2}\left(x, \dot{\dot{y}_{1}}\right)-\hat{b}_{2}\left(x, \dot{\tilde{y}}_{1}\right) \tilde{B}_{2}^{+}\left(x, \dot{\tilde{y}}_{1}\right) \tilde{C}_{2}\left(x, \dot{\tilde{y}}_{1}\right)\right) q=: \psi_{2}\left(x, q, \tilde{Y}_{2}\right) .
\end{aligned}
$$


Define as before the matrix-valued function

$$
D_{2}\left(x, \dot{\tilde{y}}_{1}\right):=\frac{\partial \psi_{2}\left(x, q, \tilde{Y}_{2}\right)}{\partial q}
$$

First assume that $D_{2} \not \equiv 0$. Then $\hat{y}_{2}^{(2)}$ explicitly depends on $q$. Again, this dependence is intrinsic in a similar sense as described above, and it can only be resolved by means of dynamic compensation if we can choose the compensator and its initial conditions in such a way that $D_{2}\left(x(t), \dot{\tilde{y}}_{1}(t)\right)=0$ for all $t$. Now note that $D_{2}$ is a function of $x$ and $\dot{\tilde{y}}_{1}$. Thus it may be possible to find a neighborhood $U$ of $x_{0}$ in $\mathscr{X}$ such that for all $x \in U$ there is a $\dot{\tilde{y}}_{1} \in \mathbb{R}^{\rho_{1}}$ such that $D_{2}\left(x, \dot{\tilde{y}}_{1}\right)=0$. By the implicit function theorem, this is the case if and only if

$$
\operatorname{rank} \frac{\partial \hat{D}_{2}}{\partial \dot{\tilde{y}}_{1}}=\operatorname{rank}\left(\frac{\partial \hat{D}_{2}}{\partial x} \frac{\partial \hat{D}_{2}}{\partial \dot{\tilde{y}}_{1}}\right)
$$

where $\hat{D}_{2}\left(x, \dot{\tilde{y}}_{1}\right)$ is the vector of functions consisting of the nonzero entries of $D_{2}$. If (24) holds, we can locally treat $x$ as a "free variable," i.e., there is a partition $\left(\dot{\tilde{y}}_{11}, \dot{\tilde{y}}_{12}\right)$ of $\dot{\tilde{y}}_{1}$ and a function $\bar{D}_{2}\left(x, \dot{\tilde{y}}_{12}\right)$ such that locally

$$
\hat{D}_{2}\left(x, \dot{\tilde{y}}_{1}\right)=0 \quad \Leftrightarrow \quad \dot{\tilde{y}}_{11}=\bar{D}_{2}\left(x, \dot{\tilde{y}}_{12}\right) \text {. }
$$

We now proceed by repeating the above procedure under the constraint that $D_{2}$ and its time derivatives are zero. If again an explicit $q$-dependence of the type described above or an explicit $q$-dependence of a time derivative of $D_{2}$ occurs, we add the function characterizing the dependence to the set of constraint functions and start all over again with the new set of constraint functions.

If, on the other hand, we have that $D_{2} \equiv 0$, we continue with subsequent steps of Singh's algorithm until an intrinsic $q$-dependence occurs and then go through the whole procedure as described above. While going through the procedure, we should apply two intermediate checks. First, it may occur at a certain step that the submanifold on which the constraints are satisfied is empty. In this case the nDDDPdm is not solvable, and we can stop the procedure. Second, assume that at a certain step the set of constraint functions is given by $\Psi_{k}\left(x, \tilde{Y}_{k}\right)$. Then, to guarantee solvability of the nDDDPdm on a neighborhood of $x_{0}$ in $\mathscr{X}$, we must necessarily have that there is a neighborhood $U$ of $x_{0}$ in $\mathscr{X}$ such that for every $x \in U$ there is a $\tilde{Y}_{k}$ satisfying $\Psi_{k}\left(x, \tilde{Y}_{k}\right)=$ 0 . By the implicit function theorem, this is the case if and only if

$$
\operatorname{rank} \frac{\partial \Psi_{k}}{\partial \tilde{Y}_{k}}=\operatorname{rank}\left(\frac{\partial \Psi_{k}}{\partial x} \frac{\partial \Psi_{k}}{\partial \tilde{Y}_{k}}\right),
$$

so that again, locally, $x$ can be treated as a free variable. Thus, if (26) does not hold, the nDDDPdm is not solvable, and we can stop the procedure.

If we want to solve the nDDDP instead of the nDDDPdm, the procedure described above follows the same lines, with a few minor differences. Namely, while solving the nDDDPdm, at every step $k$ we may resolve the intrinsic dependence of $\tilde{y}_{1}, \cdots, \tilde{y}_{k}$ on $q$ by choosing an appropriate input $u$ depending on $x, q$ and $\tilde{y}_{1}, \cdots, \tilde{y}_{k}$ and its time derivatives (see (16), (17), (21)). However, if we want to solve the nDDDP, we can only resolve the intrinsic $q$-dependence of $\tilde{y}_{1}, \cdots, \tilde{y}_{k}$ if the functions characterizing the dependence are constrained to be zero for all $t$, since $u$ is not allowed to depend on $q$. For the procedure this means that, while solving the nDDDP, we should define $D_{1}(x)=(\partial \dot{y} / \partial q)(x), D_{2}\left(x, \dot{\tilde{y}}_{1}\right)=\partial \dot{\hat{y}}_{1} / \partial q$, etc. 
Summarizing, the algorithm we are going to present will consist of applying steps of Singh's algorithm and the clamped dynamics algorithm to $\Sigma$, together with some intermediate checks. The use of Singh's algorithm can be circumvented by observing that applying Singh's algorithm to $\Sigma$ is equivalent to applying the clamped dynamics algorithm to the augmented system (cf. [35])

$$
\Sigma_{a 0}\left\{\begin{aligned}
\dot{x} & =f(x)+g(x) u+p(x) q \\
\dot{w}_{i 1} & =w_{i 2} \\
\dot{w}_{i 2} & =w_{i 3} \\
& \vdots \\
\dot{w}_{i \nu_{0}} & =v_{i} \\
y_{0 i} & =h_{i}(x)-w_{i 1}
\end{aligned} \quad(i=1, \cdots, p)\right.
$$

with $\nu_{0}:=n$. Thus we only have to use the clamped dynamics algorithm.

This leads to the following algorithm.

Algorithm 3.8 .

- Step 0

Define the system $\Sigma_{a 0}$ as in (27).

- Step $k+1$

Let $\Sigma_{a 0}, \cdots, \Sigma_{a k}$ and $\nu_{0}, \cdots, \nu_{k}$ be defined. If we apply the clamped dynamics algorithm to $\Sigma_{a k}$, where we consider $q$ as a parameter, we obtain (vectors of) functions $\phi_{1}^{k}(x, q, w), \cdots, \phi_{\pi_{k}}^{k}(x, q, w)$, where $\pi_{k}$ denotes the final step of the clamped dynamics algorithm applied to $\Sigma_{a k}$. While solving the nDDDPdm, let $\tau_{k}$ be the smallest integer for which

$$
0 \not \equiv D_{\tau_{k}}^{k}(x, w):=\frac{\partial \phi_{\tau_{k}+1}^{k}}{\partial q}
$$

While solving the nDDDP, let $\tau_{k}$ be the smallest integer for which

$$
0 \not \equiv D_{\tau_{k}}^{k}(x, w):=\frac{\partial \dot{\phi}_{\tau_{k}}^{k}}{\partial q} \text {. }
$$

If it turns out that $\tau_{k} \geqq \pi_{k}$, we set $\tau_{k}:=\pi_{k}$. Now distinguish the following cases (which should be checked sequentially):

1. $N_{\tau_{k}}^{k}:=\left\{(x, w) \mid \phi_{1}^{k}(x, w)=\cdots=\phi_{\tau_{k}}^{k}(x, w)=0\right\}=\varnothing$. In this case we stop.

2. $\operatorname{rank}\left(\partial \phi_{\tau_{k}}^{k} / \partial w\right)<\operatorname{rank}\left(\left(\partial \phi_{\tau_{k}}^{k} / \partial x\right)\left(\partial \phi_{\tau_{k}}^{k} / \partial w\right)\right)$. In this case we stop.

3. $\pi_{k}=\tau_{k}$. In this case we stop, defining $N^{*}:=N_{\pi_{k}}^{k}$.

4. If none of the cases 1,2 , or 3 hold, we define $\nu_{k+1}:=\nu_{k}+\tau_{k}$ and $\hat{D}_{\tau_{k}}^{k}$ a vector of functions consisting of a maximal number of independent entries of $D_{\tau_{k}}^{k}$. Then define the system

$$
\Sigma_{a k+1}\left\{\begin{array}{rl}
\dot{x} & =f(x)+g(x) u+p(x) q \\
\dot{w}_{i 1} & =w_{i 2} \\
\dot{w}_{i 2} & =w_{i 3} \\
\vdots & \\
\dot{w}_{i \nu_{k+1}} & =v_{i} \\
y_{k+1} & =\left(\begin{array}{l}
\phi_{\tau_{k}}^{k} \\
\hat{D}_{\tau_{k}}^{k}
\end{array}\right)
\end{array} \quad(i=1, \cdots, p) .\right.
$$

Note that at every step $k$ of Algorithm 3.8 we must apply only $\tau_{k}$ steps of the clamped dynamics algorithm to $\Sigma_{a k-1}$. If for a given $x_{0}$ at every step $k$ there is a $w_{0}^{k}$ 
such that $\left(x_{0}, w_{0}^{k}\right)$ is a regular point for the first $\tau_{k}$ steps of the clamped dynamics algorithm applied to $\Sigma_{a k-1}$, we call $x_{0}$ a regular point for Algorithm 3.8. If $x_{0}$ is a regular point, the algorithm terminates in a finite number, say $k^{*}$, of steps. Now we have the following theorem.

THEOREM 3.9. Consider the system $\Sigma$. Let $x_{0}$ be a regular point for Algorithm 3.8 applied to $\Sigma$. Then

1. The (regular) DDDPdm is locally solvable around $x_{0}$ if and only if $k^{*}=1$. Moreover, if $k^{*}=1$, the algorithm terminates because of case 3 .

2. If $k^{*}>1$, the $\mathrm{nDDDPdm}$ is locally solvable around $x_{0}$ if and only if the algorithm terminates because of case 3.

Proof. 1. See [17].

2. We only give the proof for the nDDDP. The proof for the nDDDPdm is analogous, with a few differences concerning the $q$-dependence of the compensator.

Sufficiency. Assume that the algorithm terminates because of case 3. For brevity of notation, we denote

$$
\Phi(x, w):=\phi_{\pi_{k^{*}-1}}^{k^{*}-1}(x, w) .
$$

Thus, $N^{*}=\{(x, w) \mid \Phi(x, w)=0\}$ and $N^{*}$ is a controlled invariant submanifold for $\Sigma_{a k^{*}-1}$. Since case 2 does not apply for $\Phi$, by the implicit function theorem we can find a partition $\left(w_{1}, w_{2}\right)$ of $w$ and a function $\Psi\left(x, w_{2}\right)$ such that locally $N^{*}$ can be expressed as $\left\{(x, w) \mid w_{1}=\Psi\left(x, w_{2}\right)\right\}$. Let $U \subset \mathscr{X}$ be a neighborhood of $x_{0}$ on which this parametrization of $N^{*}$ holds. Now let $u=\alpha^{*}(x, w)$ be a control that renders $N^{*}$ invariant for $\Sigma_{a k^{*}-1}$. Then it is obvious that if we apply this control to $\Sigma_{a k^{*}-1}$ and we choose our initial conditions on $N^{*}$, we will have $y_{i}^{(j)}(t)=w_{i j}(t)$ for all $t$ and thus $y(t), \cdots, y^{(n)}(t)$ are independent of $q$ for all $t$. Now let $z_{i}(i=1, \cdots, p)$ be a vector of dimension $\nu_{k^{*}-1}$ and consider the compensator

$$
Q\left\{\begin{array}{l}
\dot{z}_{i}=A z_{i}+b v_{i}(i=1, \cdots, p) \\
u=\alpha^{*}(x, z)
\end{array}\right.
$$

with $(A, b)$ in Brunovsky form, initialized at $\left(\bar{x}, \Psi\left(\bar{x}, z^{2}\right), z^{2}\right)$ for an $\bar{x} \in U$. Then from the above it is clear that this compensator locally solves the nDDDP for $\Sigma$.

Necessity. Let $x_{0}$ be a regular point for Algorithm 3.8 applied to $\Sigma$. Assume that the nDDDP is locally solvable around $x_{0}$ by means of a compensator

$$
Q\left\{\begin{array}{l}
\dot{z}=\alpha(x, z) \\
u=\gamma(x, z)
\end{array}\right.
$$

Consider the system

$$
\bar{\Sigma}_{a 0}\left\{\begin{array}{rl}
\dot{x} & =f(x)+g(x) u+p(x) q \\
\dot{w}_{i 1} & =w_{i 2} \\
\dot{w}_{i 2} & =w_{i 3} \\
\vdots & \\
\dot{w}_{i \nu_{k^{*}-1}} & =v_{i} \\
\bar{y}_{0 i} & =h_{i}(x)-w_{i 1}
\end{array} \quad(i=1, \cdots, p) .\right.
$$

Note that $\bar{\Sigma}_{a 0}$ only differs from $\Sigma_{a 0}$ in the the number of $w_{i j}$ 's for $\bar{\Sigma}_{a 0}$ is larger. Since $Q$ solves the nDDDP for $\Sigma$, it also solves the nDDDP for $\bar{\Sigma}_{a 0}$. Thus, the clamped dynamics manifold of $\bar{\Sigma}_{a 0}{ }^{\circ} Q$ is given by $M=\{(x, w, z) \mid \Phi(x, z)-w=0\}$ for some 
vector of functions $\Phi(x, z)$. Denote the clamped dynamics manifold of $\Sigma_{a k} \circ Q(k=$ $\left.0, \cdots, k^{*}-1\right)$ by $N^{k}$. By Proposition 2.5, the functions determining the clamped dynamics manifold of $\Sigma_{a k}$ are zero on $N^{k}$. This implies in particular that the functions determining the clamped dynamics manifold of $\Sigma_{a 0}$ are zero on $M$. Since $k^{*}>1$, $\tau_{0}<\pi_{0}$. Moreover, since $\partial \dot{\phi}_{\tau_{0}}^{0} / \partial q \neq 0$ and

$$
\dot{\phi}_{\tau_{0}}^{0}=\frac{\partial \phi_{\tau_{0}}^{0}}{\partial x}[f(x)+g(x) u+p(x) q]+\frac{\partial \phi_{\tau_{0}}^{0}}{\partial w} \dot{w}
$$

for $\bar{\Sigma}_{a 0}$, it is easily seen that at least one of the functions determining $M$ explicitly depends on $q$, and/or $\gamma$ depends on $q$, unless $\hat{D}_{\tau_{0}}^{0}(x, w)$ and all of its time derivatives are zero on $M$. Since the former would be in contradiction with the form of $M$ and/or the form of $Q$, the latter is the case. Now let the systems $\bar{\Sigma}_{a k}\left(k=1, \cdots, k^{*}-1\right)$ be obtained from $\bar{\Sigma}_{a k-1}$ by augmenting the outputs according to

$$
\bar{y}_{k}=\left(\begin{array}{c}
\phi_{\tau_{k-1}}^{k-1} \\
\hat{D}_{\tau_{k-1}}^{k-1}
\end{array}\right) .
$$

Again, note that $\bar{\Sigma}_{a k}$ only differs from $\Sigma_{a k}$ in that the number of $w_{i j}$ 's for $\bar{\Sigma}_{a k}$ is larger. Then, using the above and an induction argument, we can prove that the clamped dynamics manifold of $\bar{\Sigma}_{a k} \circ Q\left(k=0, \cdots, k^{*}-1\right)$ is equal to $M$. If we compare the form of $\Sigma_{a k}$ and $\bar{\Sigma}_{a k}$, we see that this implies that also $N^{k}\left(k=0, \cdots, k^{*}-1\right)$ is of the form $N^{k}=\left\{(x, w, z) \mid \Phi_{k}(x, z)-w=0\right\}$ for some vector of functions $\Phi_{k}(x, z)$. Consider the sets $\tilde{N}^{k}:=\left\{(x, w) \mid\right.$ there exists $z$ such that $\left.(x, w, z) \in N^{k}\right\}$. By the form of $N^{k}$ it is clear that $\tilde{N}^{k} \neq \varnothing$. Now, by Proposition $2.5, N^{k}$ can alternatively be written as $N^{k}=\left\{(x, w, z) \mid \phi_{\tau_{k}}^{k}(x, w)=0, \psi_{k}(x, w, z)=0\right\}$ for some vector of functions $\psi_{k}(x, w, z)$. Thus any element $(x, w) \in \tilde{N}^{k}$ must satisfy $\phi_{\tau_{k}}^{k}(x, w)=0$, which implies that $\tilde{N}^{k} \subset N_{\tau_{k}}^{k}$. Hence $N_{\tau_{k}}^{k} \neq \varnothing$ and thus the algorithm cannot terminate because of case 1 . Now assume that the algorithm terminates because of case 2. This implies that we cannot find a partition $\left(w_{1}, w_{2}\right)$ of $w$ and a vector of functions $\Psi_{k^{*}-1}\left(x, w_{2}\right)$ such that $N_{\tau_{k^{*}-1}}^{k^{*}-1}=$ $\left\{(x, w) \mid w_{1}=\Psi_{k^{*}-1}\left(x, w_{2}\right)\right\}$, i.e., $x$ cannot be a "free variable" on $N_{\tau_{k^{*}-1}}^{k^{*}-1}$. By Proposition 2.5 this implies that $x$ cannot be a free variable on $N^{k^{*}-1}$ either, which gives a contradiction with the form of $N^{k^{*}-1}$. Hence the algorithm can only terminate because of case 3 .

Remark 3.10. It is easy to see that while solving the nDDDPdm we can take the function $\alpha^{*}(x, q, w)$, which renders $N^{*}$ as an invariant manifold for $\Sigma_{a k^{*}-1}$ to be affine in $q$, i.e., we can take $\alpha^{*}$ of the form $\alpha^{*}(x, q, z)=\alpha_{1}^{*}(x, z)+\alpha_{2}^{*}(x, z) q$.

We chose the above form of the algorithm and construction of the compensator to make the algorithm and the proof of Theorem 3.9 as transparent as possible. However, the bookkeeping while applying the algorithm can become quite troublesome, and the order of the compensator can become unnecessarily high. For (relatively) simple examples, much can be improved by using ad hoc arguments in the vein of the algorithm, as will be illustrated by the following example.

Example 3.11. Consider the system

$$
\begin{array}{ll}
\dot{x}_{1}=x_{2} u_{1}+x_{4}, & y_{1}=x_{1}, \\
\dot{x}_{2}=x_{3}+q_{1}, & y_{2}=x_{3}, \\
\dot{x}_{3}=x_{1} u_{1}+x_{4}, & y_{3}=x_{5}, \\
\dot{x}_{4}=x_{5}, & y_{4}=x_{7},
\end{array}
$$


((37) cont.)

$$
\begin{aligned}
& \dot{x}_{5}=u_{2}+x_{9}, \\
& \dot{x}_{6}=x_{8} u_{3}, \\
& \dot{x}_{7}=x_{6} u_{2}+x_{8}+x_{9}+x_{6} x_{9}, \\
& \dot{x}_{8}=u_{3}, \\
& \dot{x}_{9}=x_{10}, \\
& \dot{x}_{10}=q_{2}
\end{aligned}
$$

for which we want to solve the nDDDP around points in the set $\left\{x \in \mathbb{R}^{10} \mid x_{2} \neq 0, x_{1} \neq 0\right.$, $\left.x_{6} \neq 0, x_{8} \neq 0\right\}$. Let us first restrict our attention to $y_{1}$ and $y_{2}$. We find

$$
\begin{aligned}
& \dot{y}_{1}=x_{2} u_{1}+x_{4} \Rightarrow u_{1}=\frac{1}{x_{2}}\left(\dot{y}_{1}-x_{4}\right), \\
& \dot{y}_{2}=x_{1} u_{1}+x_{4}=\frac{x_{1}}{x_{2}}\left(\dot{y}_{1}-x_{4}\right)+x_{4}, \\
& \ddot{y}_{2}=\frac{\dot{y}_{1}}{x_{2}}\left(\dot{y}_{1}-x_{4}\right)-\frac{x_{1}\left(x_{3}+q_{1}\right)}{x_{2}^{2}}\left(\dot{y}_{1}-x_{4}\right)+\frac{x_{1}}{x_{2}}\left(\ddot{y}_{1}-x_{5}\right)+x_{5} .
\end{aligned}
$$

Thus $\ddot{y}_{2}$ intrinsically depends on $q_{1}$. Hence we should have

$$
\frac{x_{1}}{x_{2}^{2}}\left(\dot{y}_{1}-x_{4}\right)=0 \text {. }
$$

Since we are working in a neighborhood of point for which $x_{1} \neq 0, x_{2} \neq 0$, this implies that $\left(1 / x_{2}\right)\left(\dot{y}_{1}-x_{4}\right)=u_{1}=0$.

Having chosen $u_{1}=0$, we see from the structure of the system $y_{1}$ and $y_{2}$ can be made independent of $q_{2}$ if and only if $y_{3}$ and $y_{4}$ can be made independent of $q_{2}$. Restricting our attention to $y_{3}$ and $y_{4}$, we find

$$
\begin{aligned}
& \dot{y}_{3}=u_{2}+x_{0} \Rightarrow u_{2}=\dot{y}_{3}-x_{9}, \\
& \dot{y}_{4}=x_{6} u_{2}+x_{8}+x_{9}+x_{6} x_{9}=x_{6} \dot{y}_{3}+x_{8}+x_{9}, \\
& \ddot{y}_{4}=x_{8} \dot{y}_{3} u_{3}+x_{6} \ddot{y}_{3}+u_{3}+x_{10} \Rightarrow u_{3}=\frac{1}{x_{8} \dot{y}_{3}+1}\left(\ddot{y}_{4}-x_{6} \ddot{y}_{3}-x_{10}\right) .
\end{aligned}
$$

Thus we can solve the nDDDPdm by choosing a compensator

$$
\begin{aligned}
\dot{z} & =v_{1}, \\
u_{1} & =0, \\
u_{2} & =z-x_{9}, \\
u_{3} & =\frac{1}{x_{8} z+1}\left(v_{2}-x_{6} v_{1}-x_{10}\right),
\end{aligned}
$$

with $z(0) \neq-\left(1 / x_{8}(0)\right)$ and $v_{1}, v_{2}$ denoting the new inputs.

4. The nonlinear model matching problem. In this section we will use the results of the previous section to give a complete local solution of the nonlinear model matching problem. For linear systems, the idea that we can obtain a solution of the nonlinear model matching problem by associating it with a disturbance decoupling problem was elaborated in [32], [11]. It was first extended to nonlinear systems in [10]. 
First, we give a formulation of the problem. Consider a nonlinear plant $P$, described by equations of the form

$$
P\left\{\begin{array}{l}
\dot{x}=f(x)+g(x) u \\
y=h(x)
\end{array}\right.
$$

where $x \in \mathscr{X}$, an open subset of $\mathbb{R}^{n}$, the inputs $u \in \mathbb{R}^{m}$, the outputs $y \in \mathbb{R}^{p}, f$ and $h$ are vector-valued analytic functions, and $g$ is a matrix-valued analytic function, all of appropriate dimensions.

Furthermore, let a nonlinear model $M$ be given, which is described by the equations

$$
M\left\{\begin{array}{l}
\dot{x}^{M}=f^{M}\left(x^{M}\right)+g^{M}\left(x^{M}\right) u^{M} \\
y^{M}=h^{M}\left(x^{M}\right)
\end{array}\right.
$$

where $x^{M} \in \mathscr{X}^{M}$, an open subset of $\mathbb{R}^{n_{M}}$, the inputs $u^{M} \in \mathbb{R}^{m}$, the outputs $y^{M} \in \mathbb{R}^{p}, f^{M}$ and $h^{M}$ are vector-valued analytic functions, and $g^{M}$ is a matrix-valued analytic function, all of appropriate dimensions.

The compensator $Q$ used to control $P$ is a nonlinear system described by equations of the form

$$
Q\left\{\begin{array}{l}
\dot{z}=a(x, z)+b(x, z) u^{M} \\
u=c(x, z)+d(x, z) u^{M}
\end{array}\right.
$$

with state $z \in \mathbb{R}^{\nu}$ and real analytic $a, b, c, d$.

The usual definition of the nonlinear model matching problem is given below (see [6], [10]).

DEFINITION 4.12 (Nonlinear model matching problem (MMP)). Given a plant $P=(f, g, h)$, a model $M=\left(f^{M}, g^{M}, h^{M}\right)$, and a point $\left(x_{0}, x_{0}^{M}\right) \in \mathscr{X} \times \mathscr{X}^{M} \subset \mathbb{R}^{n} \times \mathbb{R}^{n_{M}}$, find neighborhoods $U$ of $x_{0}$ and $U^{M}$ of $x_{0}^{M}$, an integer $\nu$, an open subset $V$ of $\mathbb{R}^{\nu}$, a compensator $Q=(a, b, c, d)$ with $a, b, c, d$ real analytic functions defined on $V \times U$, and a map $F: U \times U^{M} \mapsto V$, with the property that

$$
y^{P \circ Q}\left(x, F\left(x, x^{M}\right), t\right)-y^{M}\left(x^{M}, t\right)
$$

is independent of $u^{M}$ for all $t$ and for all $\left(x, x^{M}\right) \in U \times U^{M}$.

Given $M$ and $P$ we define an extended system $E$ :

$$
E\left\{\begin{array}{l}
\dot{x}^{E}=f^{E}\left(x^{E}\right)+g^{E}\left(x^{E}\right) u^{E}+p^{E}\left(x^{E}\right) q^{E} \\
y^{E}=h^{E}\left(x^{E}\right)
\end{array}\right.
$$

with

$$
\begin{gathered}
x^{E}=\left(\begin{array}{c}
x \\
x^{M}
\end{array}\right), \quad f^{E}\left(x^{E}\right)=\left(\begin{array}{c}
f(x) \\
f^{M}\left(x^{M}\right)
\end{array}\right), \quad g^{E}\left(x^{E}\right)=\left(\begin{array}{c}
g(x) \\
0
\end{array}\right), \\
p^{E}\left(x^{E}\right)=\left(\begin{array}{c}
0 \\
g^{M}\left(x^{M}\right)
\end{array}\right), \quad h^{E}\left(x^{E}\right)=h(x)-h^{M}\left(x^{M}\right) .
\end{gathered}
$$

THEOREM 4.13. The MMP is solvable for $(M, P)$ if and only if the $\mathrm{nDDDPdm}$ is solvable for $E$.

Proof. Necessity. Assume that the MMP is solvable for $(M, P)$ by means of a compensator $Q$ of the form (43). Then from the definition of the MMP it is obvious that the compensator

$$
Q_{E}\left\{\begin{array}{l}
\dot{z}=\alpha\left(x^{E}, q^{E}, z\right) \\
u=\gamma\left(x^{E}, q^{E}, z\right)
\end{array}\right.
$$

with $\alpha\left(x^{E}, q^{E}, z\right)=a(x, z)+b(x, z) q^{E}, \quad \gamma\left(x^{E}, q^{E}, z\right)=c(x, z)+d(x, z) q^{E}$ solves the nDDDPdm for $E$. 
Sufficiency (see also [10]). Assume that the nDDDPdm is solvable for $E$ by means of a compensator

$$
Q_{E}\left\{\begin{array}{l}
\dot{z}=\alpha_{1}\left(x^{E}, z\right)+\alpha_{2}\left(x^{E}, z\right) q^{E} \\
u=\gamma_{1}\left(x^{E}, z\right)+\gamma_{2}\left(x^{E}, z\right) q^{E} .
\end{array}\right.
$$

Note that we can indeed take the compensator to be affine in $q$ (see Remark 3.10).

Consider the following compensator for $P$ :

$$
Q\left\{\begin{array}{l}
\dot{z}_{1}=f^{M}\left(z_{1}\right)+g^{M}\left(z_{1}\right) u^{M} \\
\dot{z}_{2}=\alpha_{1}\left(x, z_{1}, z_{2}\right)+\alpha_{2}\left(x, z_{1}, z_{2}\right) u^{M} \\
u=\gamma_{1}\left(x, z_{1}, z_{2}\right)+\gamma_{2}\left(x, z_{1}, z_{2}\right) u^{M} .
\end{array}\right.
$$

Then it is easy to see that $Q$ solves the MMP for $(M, P)$.

Thus, using the results from Theorem 3.9, we can check if the MMP is solvable for given $(M, P)$ and we can construct a compensator that solves the MMP. We will illustrate this by means of an example.

Example 4.14. Consider the plant

$$
P \begin{cases}\dot{x}_{1}=x_{2}+x_{2} u_{2} & y_{1}=x_{1} \\ \dot{x}_{2}=u_{1} & y_{2}=x_{3} \\ \dot{x}_{3}=u_{2} & y_{3}=x_{3}\end{cases}
$$

and the model

$$
M \begin{cases}\dot{x}_{1}^{M}=x_{2}^{M} & y_{1}^{M}=x_{1}^{M} \\ \dot{x}_{2}^{M}=x_{3}^{M}+u_{1}^{M} & y_{2}^{M}=x_{2}^{M} \\ \dot{x}_{3}^{M}=u_{2}^{M} & y_{3}^{M}=x_{4}^{M} \\ \dot{x}_{4}^{M}=-x_{4}^{M} & \end{cases}
$$

Then

$$
\begin{aligned}
& \dot{y}_{1}-\dot{y}_{1}^{M}=x_{2}+x_{2} u_{2}=x_{2}\left(1+\dot{y}_{3}-\dot{y}_{3}^{M}-x_{4}^{M}\right)-x_{2}^{M}, \\
& \dot{y}_{2}-\dot{y}_{2}^{M}=u_{1}-x_{3}^{M}-u_{1}^{M} \Rightarrow u_{1}=\dot{y}_{2}-\dot{y}_{2}^{M}+x_{3}^{M}+u_{1}^{M}, \\
& \dot{y}_{3}-\dot{y}_{3}^{M}=u_{2}+x_{4}^{M} \Rightarrow u_{2}=\dot{y}_{3}-\dot{y}_{3}^{M}-x_{4}^{M}, \\
& \ddot{y}_{1}-\ddot{y}_{1}^{M}=\left(\dot{y}_{2}-\dot{y}_{2}^{M}+x_{3}^{M}+u_{1}^{M}\right)\left(1+\dot{y}_{3}-\dot{y}_{3}^{M}-x_{4}^{M}\right)+x_{2}\left(\ddot{y}_{3}-\ddot{y}_{3}^{M}-x_{4}^{M}\right)-x_{3}^{M}-u_{1}^{M} .
\end{aligned}
$$

Thus, $\ddot{y}_{1}-\ddot{y}_{1}^{M}$ intrinsically depends on $u_{1}^{M}$ (the disturbances). Hence to solve the MMP we must have

$$
\dot{y}_{3}-\dot{y}_{3}^{M}-x_{4}^{M}=u_{2}=0 \text {. }
$$

Then it is easy to see that we can solve the MMP by means of the nonregular compensator

$$
Q\left\{\begin{array}{l}
\dot{z}_{1}=z_{2} \\
\dot{z}_{2}=z_{3}+u_{1}^{M} \\
\dot{z}_{3}=u_{2}^{M} \\
\dot{z}_{4}=-z_{4} \\
u_{1}=z_{3}+u_{1}^{M} \\
u_{2}=0
\end{array}\right.
$$

with $z(0)=x^{M}(0)$.

5. Conclusions and remarks. In this paper we formulated and solved the local nDDDP. The solution was given by means of a constructive algorithm. A drawback 
of the algorithm presented in this paper is that it does not give a compensator of minimal order solving the nDDDPdm. Therefore we cannot make any statement about the solvability of the DDP by means of nonregular static state feedback. This remains a problem for future research. Another topic for future research is the generalization of the theory developed in this paper to general nonlinear systems of the form

$$
\left\{\begin{array}{l}
\dot{x}=f(x, q, u) \\
y=h(x, u) .
\end{array}\right.
$$

Using the algorithm given in this paper we can immediately give sufficient conditions for the solvability of the nDDDPdm for (53). Namely, consider the following extended system obtained from (53):

$$
\left\{\begin{array}{l}
\dot{x}=f(x, q, u) \\
\dot{u}=v \\
y=h(x, u) .
\end{array}\right.
$$

Then any solution to the nDDDPdm for (53) gives rise to a solution of the problem for (53). The converse is easily seen to be false. However, since for the system (53) versions of Singh's algorithm and the clamped dynamics algorithm are also available (see [26], [23], [24] for Singh's algorithm and [35] for the clamped dynamics algorithm), it seems that the method of this paper can be straightforwardly extended to systems of the form (53).

By proving that the solvability of the nonlinear MMP is equivalent to the solvability of an associated nDDDP with disturbance measurements, we also established a complete local solution of this problem. A problem that remains unsolved is the problem of internal stability of the compensated plant after we have solved the nonlinear MMP. Until now this problem has only been addressed in [3] in the case where the plant is a single-input, single-output system and in [15] in the case where the plant is decouplable by static state feedback. The problem consists in the fact that, even if we start from an internally stable plant and an internally stable model, we may very well introduce unstable unobservable modes in the closed loop. To solve this problem, further investigation of the structure of a model matching configuration is needed, especially concerning the "fixed" and "free" modes of such a configuration. For linear systems this investigation has already been performed in [31]. For nonlinear systems this is undoubtedly much more difficult to answer. So far, only results about "fixed" modes in the solution of the input-output decoupling problem have been obtained in [12]. It is not clear if a similar analysis is applicable for the nonlinear MMP considered here. We leave this open for future research.

\section{Appendix.}

Proof of Proposition 2.5. Denote the submanifolds obtained while applying the clamped dynamics algorithm to (6) and (6), (10) by $N_{k}\left(=\left\{x \mid \phi_{k}(x)=0\right\}\right), M_{k}$, respectively. We will prove by induction that we can find vectors of functions $\psi_{k}(x, z)$ such that $M_{k}=\left\{(x, z) \mid \xi_{k}(x, z)=0\right\}$, with

$$
\xi_{k}(x, z)=\left(\begin{array}{c}
\phi_{k}(x) \\
\psi_{k}(x, z)
\end{array}\right) .
$$

Obviously, we have $N_{0}=\left\{x \mid \phi_{0}(x)=0\right\}, \quad M_{0}=\left\{(x, z) \mid \xi_{0}(x, z)=0\right\}$, with $\phi_{0}(x)=$ $\xi_{0}(x, z)=h(x)$. Hence our claim holds for $k=0$. Now apply the first step of the clamped 
dynamics algorithm to (6), yielding matrices $A_{1}(x), B_{1}(x)$, a vector of functions $\phi_{1}(x)$, and $N_{1}=\left\{x \mid \phi_{1}(x)=0\right\}$. Applying the first step of the clamped dynamics algorithm to (6), (10) yields

$$
\begin{aligned}
\dot{\xi}_{0}(x, z) & =\frac{\partial \phi_{0}}{\partial x}(x)[f(x)+g(x)(\gamma(x, z)+\delta(x, z) v)] \\
& =A_{1}(x)+B_{1}(x) \gamma(x, z)+B_{1}(x) \delta(x, z) v .
\end{aligned}
$$

Since $\left(x_{0}, z_{0}\right)$ is a regular point for the clamped dynamics algorithm applied to (6), (10), $B_{1}(x) \delta(x, z)$ has full rank $\tilde{r}_{1}$ in a neighborhood of $\left(x_{0}, z_{0}\right)$ in $M_{0}$. It is clear that $\tilde{r}_{1}<r_{1}=$ rank $B_{1}(x)$. Moreover, the rows of $\hat{B}_{1}(x) \delta(x, z)$ are linearly dependent on the rows of $\tilde{B}_{1}(x) \delta(x, z)$, since the rows of $\hat{B}_{1}(x)$ are linearly dependent on the rows of $\tilde{B}_{1}(x)$. Thus we can permute the entries of $\xi_{0}(x, z)$ in such a way that the first $\tilde{r}_{1}$ rows of $B_{1}(x) \delta(x, z)$ are linearly independent and the last $\left(p_{0}-r_{1}\right)$ entries of $\xi_{0}(x, z)$ consist of $\hat{\phi}_{0}(x)$, i.e., we can write $(55)$ as

$$
\left(\begin{array}{c}
\dot{\tilde{\sigma}}_{0}(x, z) \\
\dot{\hat{\sigma}}_{0}(x, z) \\
\dot{\hat{\phi}}_{0}(x, z)
\end{array}\right)=\left(\begin{array}{c}
\tilde{A}_{\sigma 1}(x)+\tilde{B}_{\sigma 1}(x) \gamma(x, z) \\
\hat{A}_{\sigma 1}(x)+\hat{B}_{\sigma 1}(x) \gamma(x, z) \\
\hat{A}_{1}(x)+\hat{B}_{1}(x) \gamma(x, z)
\end{array}\right)+\left(\begin{array}{c}
\tilde{B}_{\sigma 1}(x) \delta(x, z) \\
\hat{B}_{\sigma 1}(x) \delta(x, z) \\
\hat{B}_{1}(x) \delta(x, z)
\end{array}\right) v
$$

where $\left(\tilde{\sigma}_{0}^{T}, \hat{\sigma}_{0}^{T}\right)^{T}$ consists of the entries of $\tilde{\phi}_{0}$ and $\tilde{B}_{\sigma 1}(x) \delta(x, z)$ has full row rank $\tilde{r}_{1}$ in a neighborhood of $\left(x_{0}, z_{0}\right)$ in $M_{0}$. Observe that

$$
\left(\begin{array}{c}
\tilde{B}_{\sigma 1}(x) \\
\hat{B}_{\sigma 1}(x)
\end{array}\right)
$$

has full row rank $r_{1}$ in a neighborhood of $\left(x_{0}, z_{0}\right)$ in $M_{0}$. Thus, we can rewrite $\dot{\phi}_{\hat{0}}(x, z)$ in $(56)$ as

$$
\dot{\hat{\phi}}_{0}(x, z)=\hat{A}_{1}(x)+\hat{B}_{1}(x)\left(\begin{array}{c}
\tilde{B}_{\sigma 1}(x) \\
\hat{B}_{\sigma 1}(x)
\end{array}\right)^{+}\left[\left(\begin{array}{c}
\dot{\tilde{\sigma}}_{0}(x, z) \\
\dot{\hat{\sigma}}(x, z)
\end{array}\right)-\left(\begin{array}{c}
\tilde{A}_{\sigma 1}(x) \\
\hat{A}_{\sigma 1}(x)
\end{array}\right)\right],
$$

where

$$
\left(\begin{array}{c}
\tilde{B}_{\sigma 1} \\
\hat{B}_{\sigma 1}
\end{array}\right)^{+} \text {is a right inverse of }\left(\begin{array}{c}
\tilde{B}_{\sigma 1} \\
\hat{B}_{\sigma 1}
\end{array}\right) .
$$

Let $\left(\tilde{B}_{\sigma 1}(x) \delta(x, z)\right)^{+}$be a right inverse of $\tilde{B}_{\sigma 1}(x) \delta(x, z)$. Letting $v=$ $-\left(\tilde{B}_{\sigma 1}(x) \delta(x, z)\right)^{+} \tilde{A}_{\sigma 1}(x)$ we find from (56)

$$
\left(\begin{array}{c}
\dot{\tilde{\sigma}}_{0}(x, z) \\
\dot{\hat{\sigma}}_{0}(x, z)
\end{array}\right)=\left(\begin{array}{c}
0 \\
\hat{A}_{\sigma 1}(x)-\hat{B}_{\sigma 1}(x)\left(\tilde{B}_{\sigma 1}(x) \delta(x, z)\right)^{+} \tilde{A}_{\sigma 1}(x)
\end{array}\right)=:\left(\begin{array}{c}
0 \\
\bar{\sigma}_{1}(x, z)
\end{array}\right) .
$$

Then $M_{1}$ is given as $M_{1}=\left\{(x, z) \mid \phi_{0}(x)=0, \dot{\hat{\phi}}_{0}(x, z)=0, \bar{\sigma}_{1}(x, z)=0\right\}$. Since $\dot{\tilde{\sigma}}_{0}(x, z)=0$ on $M_{1}$ and (obviously)

$$
\left(\begin{array}{c}
\tilde{B}_{\sigma 1}(x) \\
\hat{B}_{\sigma 1}(x)
\end{array}\right)^{+}\left(\begin{array}{c}
\tilde{A}_{\sigma 1}(x) \\
\hat{A}_{\sigma 1}(x)
\end{array}\right)=\tilde{B}_{1}^{+}(x) \tilde{A}_{1}(x)
$$

we find from (57) that on $M_{1}$

$$
\dot{\hat{\phi}}_{0}(x, z)=\hat{A}_{1}(x)-\hat{B}_{1}(x) \tilde{B}_{1}^{+}(x) \tilde{A}_{1}(x)=\hat{\phi}_{1}(x) .
$$

Thus, $M_{1}=\left\{(x, z) \mid \phi_{0}(x)=0, \bar{\phi}_{1}(x)=0, \bar{\sigma}_{1}(x, z)=0\right\}$. Assume that $\left(\bar{\phi}_{1}^{T}(x), \bar{\sigma}_{1}^{T}(x, z)\right)^{T}$ has constant rank $\tilde{s}_{1}$ in a neighborhood of $\left(x_{0}, z_{0}\right)$ in $M_{1}$. Obviously, $\tilde{s}_{1}>s_{1}$. Since $\bar{\phi}_{1}(x)$ has constant rank $s_{1}$ in a neighborhood of $\left(x_{0}, z_{0}\right)$ in $M_{1}$, we can permute the entries of $\bar{\phi}_{1}(x)$ and $\bar{\sigma}_{1}(x, z)$ such that $\left(\bar{\phi}_{11}(x), \cdots, \bar{\phi}_{1 s_{1}}(x), \bar{\sigma}_{11}(x, z), \cdots, \bar{\sigma}_{1 \tilde{s}_{1}-s_{1}}(x, z)\right)$ 
are independent on $M_{1}$. Defining $\phi_{1}(x)=\left(\phi_{0}^{T}(x), \bar{\phi}_{11}(x), \cdots, \bar{\phi}_{1 s_{1}}(x)\right)^{T}, \psi_{1}(x, z)=$ $\left(\bar{\sigma}_{11}(x, z), \cdots, \bar{\sigma}_{1 \tilde{s}_{1}-s_{1}}(x, z)\right)^{T}$ we find $M_{1}=\left\{(x, z) \mid \phi_{1}(x)=0, \psi_{1}(x, z)=0\right\}$. Hence our claim also holds for $k=1$. Using similar arguments as above, we can prove that our claim holds for $k=0, \cdots, k^{*}$, which completes the proof.

Acknowledgments. I would like to thank Henk Nijmeijer and Arjan van der Schaft for the many useful discussions we have had on the contents and organization of this paper.

\section{REFERENCES}

[1] G. BASIle AND G. MARRo, Controlled and conditioned invariant subspaces in linear system theory, J. Optim. Theory Appl., 3 (1969), pp. 306-315.

[2] S. P. Bhattacharyya, Disturbance rejection in linear systems, Internat. J. Control, 5 (1971), pp. 633637.

[3] C. I. BYRNES, R. CASTRO, AND A. ISIDORI, Linear model matching with prescribed tracking error and internal stability for nonlinear systems, in Analysis and Optimization of Systems, A. Bensoussan and J. L. Lions, eds., Lecture Notes in Control and Inform. Sci., 111, Springer-Verlag, Berlin, 1988 , pp. 249-258.

[4] C. I. BYRNES AND A. IsIDORI, A frequency domain philosophy for nonlinear systems, with applications to stabilization and adaptive control, in Proc. 23rd CDC, Las Vegas, NV, 1984, pp. 1569-1573.

[5] —_, Local stabilization of minimum-phase nonlinear systems, Systems Control Lett., 11 (1988), pp. 9-17.

[6] M. D. Di BENEDETTO, A condition for the solvability of the nonlinear model matching problem, in New Trends in Nonlinear Control Theory, J. Descusse, M. Fliess, A. Isidori, and D. Leborgne, eds., Lecture Notes in Control and Inform. Sci., 122, Springer-Verlag, Berlin, 1988, pp. 102-115.

[7] - New results on nonlinear model matching, preprint, 1988.

[8] M. D. Di Benedetto AND J. W. GRizzle, An intrinsic notion of regularity for local output nulling, inversion and dynamic extension of nonsquare systems, preprint, 1989.

[9] M. D. Di Benedetto, J. W. GRizzle, AND C. H. MOOG, Rank invariants of nonlinear systems, SIAM J. Control Optim., 27 (1989) pp. 658-672.

[10] M. D. DI BENEDETTO AND A. IsIDORI, The matching of nonlinear models via dynamic state feedback, SIAM J. Control Optim., 18 (1986), pp. 420-436.

[11] E. EmRe AND M. L. J. HaUtus, A polynomial characterization of $(A, B)$-invariant and reachability subspaces, SIAM J. Control Optim., 18 (1980), pp. 420-436.

[12] A. IsIDORI AND J. W. GRIZZLE, Fixed modes and nonlinear noninteracting control with stability, IEEE Trans. Automat. Control, AC-33 (1988), pp. 907-914.

[13] R. M. HIRSCHORN, Invertibility of multivariable nonlinear control systems, IEEE Trans. Automat. Control, AC-24 (1979), pp. 855-865.

[14] - $(A, B)$-invariant distributions and disturbance decoupling of nonlinear systems, SIAM J. Control. Optim., 19 (1981), pp. 1-19.

[15] H. J. C. HuiJberts, Nonlinear model matching with an application to Hamiltonian systems, IFAC Nonlinear Control Systems Design Symposium 1989, Capri, Italy, preprints.

[16] _ Nonlinear model matching: A local solution and two worked examples, in Proc. 1990 American Control Conference, San Diego, CA, 1990.

[17] H. J. C. Huijberts, H. NiJmeijer, AND L. L. M. van Der Wegen, Dynamic disturbance decoupling for nonlinear systems, SIAM J. Control Optim., 30 (1992), pp. 336-349, this issue.

[18] H. J. C. HuiJBERTS AND H. NiJMEIJER, Local nonlinear model matching: From linearity to nonlinearity, Automatica, 26 (1990), pp. 973-983.

[19] A. ISIDORI, The matching of a prescribed linear input-output behavior in a nonlinear system, IEEE Trans. Automat. Control, AC-30 (1985), pp. 258-265.

[20] — Nonlinear Control Systems, 2nd ed., Springer-Verlag, Berlin, 1989.

[21] A. Isidori, A. J. Krener, C. Gori-Giorgi, AND S. Monaco, Nonlinear decoupling via feedback: A differential geometric approach, IEEE Trans. Automat. Control, AC-26 (1981), pp. 331-345.

[22] A. IsIDORI AND C. H. MOG, On the nonlinear equivalent of the notion of transmission zeros, in Modelling and Adaptive Control, C. I. Byrnes and A. Kurzhanski, eds., Lecture Notes in Control and Inform. Sci., 105, Springer-Verlag, Berlin, 1988, pp. 146-158.

[23] U. КоттА, Right inverse of a discrete-time nonlinear system, Internat. J. Control, 51 (1990), pp. 1-9. 
[24] U. KotTA AND H. NiJMeiJer, Dynamic disturbance decoupling for discrete time nonlinear systems Proc. Academy USSR, Technical Cybernetics 1991, to appear. (In Russian.)

[25] A. J. KRENER, (Adf, g), (adf, g) and locally (adf, g) invariant and controllability distributions, SIAM J. Control Optim., 23 (1985), pp. 523-549.

[26] C.-W. LI AND Y.-K. FENG, Functional reproducibility of general multivariable analytic nonlinear systems, Internat. J. Control, 45 (1987), pp. 255-268.

[27] M. MAlabre, Structure à l'infini des triplets invariants. Application à la poursuite parfaite de modèle, in Analysis and Optimization of Systems, A. Bensoussan and J. L. Lions, eds., Lecture Notes in Control and Inform. Sci., 44, Springer-Verlag, Berlin, 1982, pp. 43-53.

[28] R. MARINO, High-gain feedback in nonlinear control systems, Internat. J. Control, 42 (1985), pp. 13691385.

[29] C. H. MOOG, A. M. PERdon, AND G. CONTE, Model matching and factorization for nonlinear systems: A structural approach, SIAM J. Control Optim., 29 (1991), pp. 769-785.

[30] B. C. Moore AND L. M. Silverman, Model matching by state feedback and dynamic compensation, IEEE Trans. Automat. Control, AC-17 (1972), pp. 491-497.

[31] A. S. MORSE, Structure and design of linear model following systems, IEEE Trans. Automat. Control, AC-18 (1973), pp. 346-354.

[32] — Minimal solutions to transfer matrix equations, IEEE Trans. Automat. Control, AC-21 (1976), pp. 131-133.

[33] H. NiJmeijer And A. J. VAn Der Schaft, Nonlinear Dynamical Control Systems, Springer-Verlag, New York, 1990.

[34] S. N. SINGH, A modified algorithm for invertibility in nonlinear systems, IEEE Trans. Automat. Control, AC-26 (1990), pp. 595-598.

[35] A. J. VAN DER SCHAFT, On clamped dynamics of nonlinear systems, in Analysis and Control of Nonlinear Systems, C. I. Byrnes, C. F. Martin, and R. E. Saeks, eds., Elsevier, Amsterdam, the Netherlands, 1988, pp. 499-506.

[36] W. M. Wonham, Linear Multivariable Control: A Geometric Approach, 3rd ed., Springer-Verlag, New York, 1985. 